\title{
UN NOUVEL APPAREIL DE PÊCHE ÉLECTRIQUE PORTABLE : LE "MARTIN - PÊCHEUR"
}

\author{
par CI. GOSSET, P. LAMARQUE et Nicole CHARLON \\ avec la collaboration technique de \\ C. GARAÏCOECHEA \\ Station d'Hydrobiologie - I.N.R.A. \\ B. P. 79 - 64 - BIARRITZ
}

Le "MARTIN-PECHEUR " (Brevet C.N.R.S. *) a été spécialement conçu par la Station d'Hydrobiologie de BIARRITZ, pour les rivières à truites, (résistivité comprise entre 1000 et $40000 \mathrm{ohms}-\mathrm{cm}$ ) de manière à assurer une forte efficacité à la pêche, tout en respectant les normes de légèreté et de maniabilité qui font les qualités d'un appareil portable.

\section{1. - CARACTERISTIQUES TECHNIQUES}

\section{1 - Poids et encombrement}

Livré en boîtier duralinox, l'appareil pèse $10 \mathrm{~kg}$; en boitier de tôle, il pèse $12 \mathrm{~kg}$ (batterie comprise).

Ses dimensions sont les suivantes : $310 \times 110 \times 300 \mathrm{~mm}$.

\section{2 - Fixation (fig. 1.)}

L'appareil est attaché sur le dos par des courroies réglables, passant sur les épaules ; le boitier de commande et de contrôle est attaché sur le ventre par une ceinture.

(*) Exploité par Electro-Pullman, 5, rue des Bruyères, 92-BOURG-LA-REINE. 


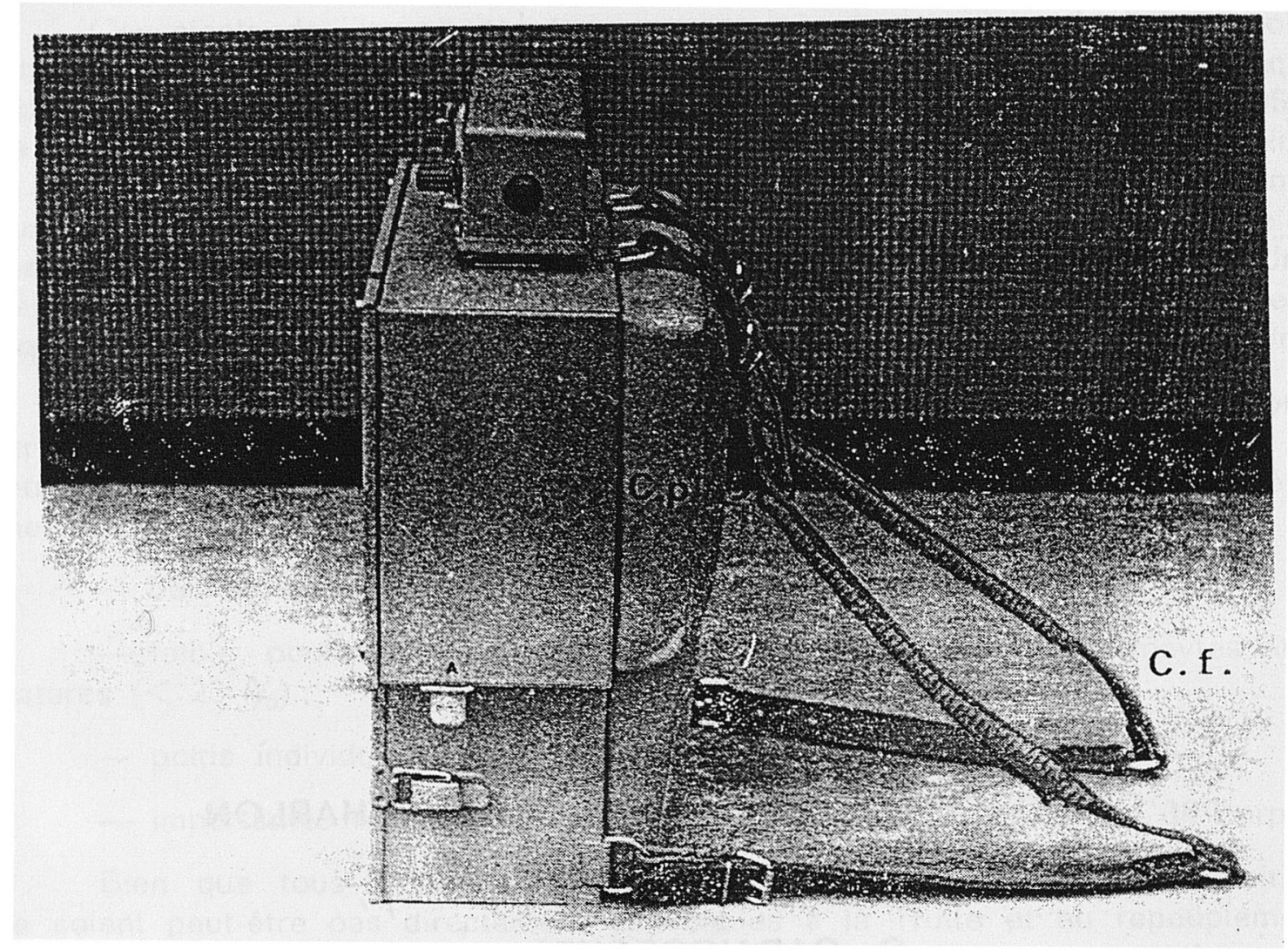

Fig. 1 - Système de fixation.

C.p. : coussin de protection.

C. f. : courroies de fixation.

\section{3 - Etanchéité}

L'appareil peut résister sans dommage aux intempéries et même supporter la chute éventuelle de l'opérateur dans la rivière.

\section{4 - Dispositif électrique (fig. 2)}

Réalisé entièrement en circuit imprimé, le montage électronique contenu dans la partie supérieure du boitier principal est complètement isolé du reste de. l'appareil.

\subsection{1 - Alimentation}

Elle est assurée par une batterie Cadmium-Nickel $12 \mathrm{~V}, 9$ Ah (l'appareil est iivré avec 2 batteries). Ce genre de batterie permet un fort régime de décharge ; il a une très grande longévité ( 1000 cycles) et l'électrolyte ne pouvant se vider, il peut être mis dans toutes les positions.

\subsection{2 - Type de courant}

Le courant utilisè est le courant en créneaux de fréquence $400 \mathrm{~Hz}$, à rapport cyclique réglable entre 10 et 50 pour cent (Fig. 3).

Ce type de courant a été choisi en raison de sa grande efficacité et de son innocuité pour le poisson, relativement à d'autres courants interrompus. Efficacitè et innocuité sont encore accrues par le choix de la fréquence de $400 \mathrm{~Hz}$, (particulièrement efficace pour les truitelles et anguilles). La possibilité de régler le rapport 


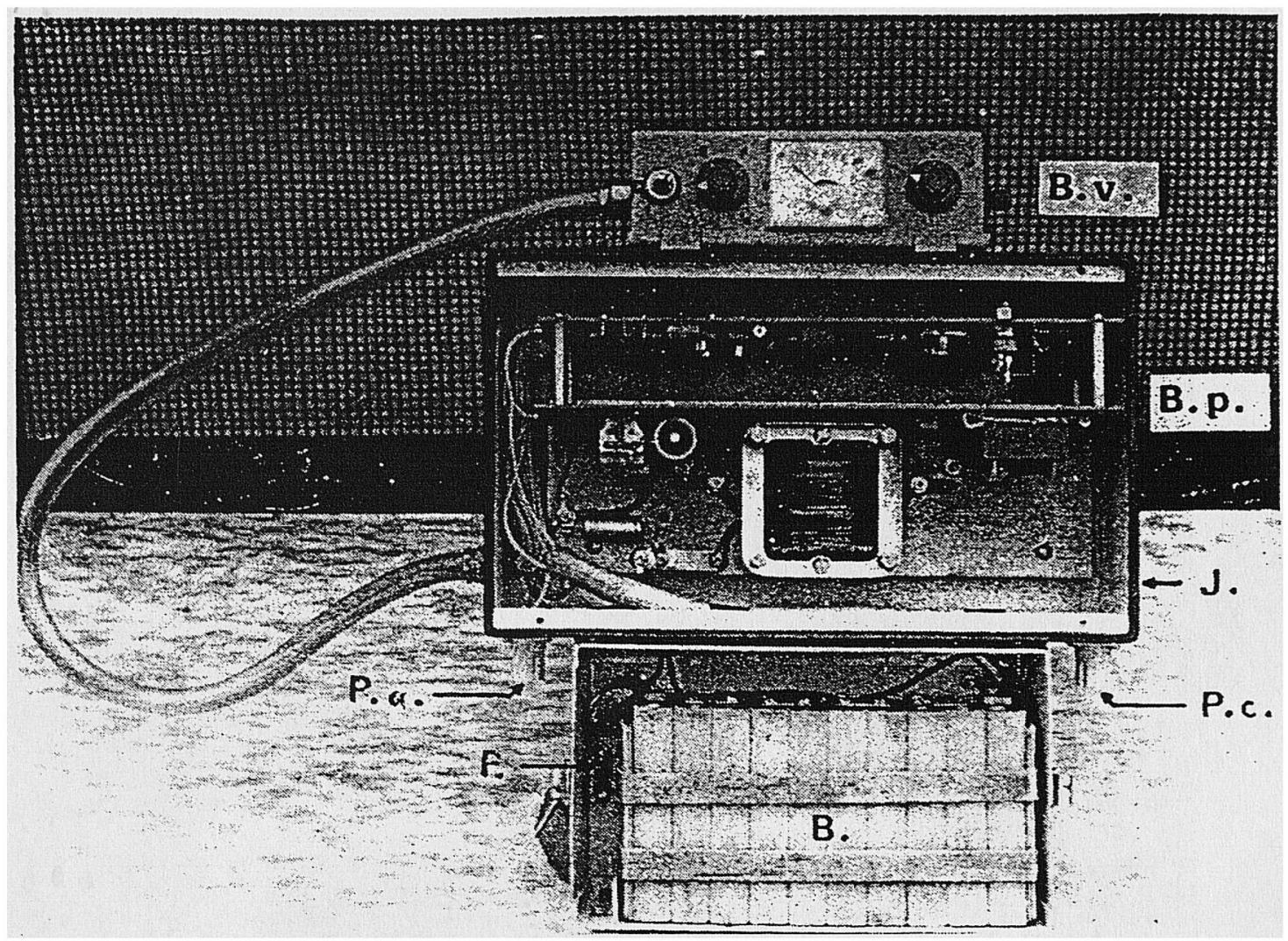

Fig. 2 - Vue d'ensemble de l'appareillage électrique du Martin-Pêcheur.

B.v. : boitier ventral de commande sur son support.

B.p. : boitier principal.

B. : batterie Cadmium-Nickel.

J. : joints d'étanchéité.

P.c. : prise de cathode.

P.a. : prise d'anode.

F. : fusible de secours.

cyclique, de manière à pêcher avec le rapport cyclique maximal, dans la limite de puissance de l'appareil, est un facteur supplémentaire d'accroissement de l'efficacité.

\subsection{3 - Tension}

Les tensions utilisées sont les suivantes : 150, 200 et 250 volts. Chaque tension correspond à une utilisation dépendant principalement de la conductivité de l'eau.

\subsection{4 - Schéma de principe (Fig. 4)}

A partir de la batterie d'accumulateurs, on alimente un convertisseur transistorisé à tension de sortie réglable. Cette tension est ensuite "découpée » par un "chopper" (rupteur) à thyristors commandé par, un multivibrateur.

\subsection{1 - Convertisseur}

Il permet d'obtenir une tension continue élevée à partir de la tension continue d'alimentation 12 volts.

Ce convertisseur utilise deux transistors de puissance montés en cscillateur. L'utilisation d'un transformateur de réaction à noyau saturé permet d'assurer à 

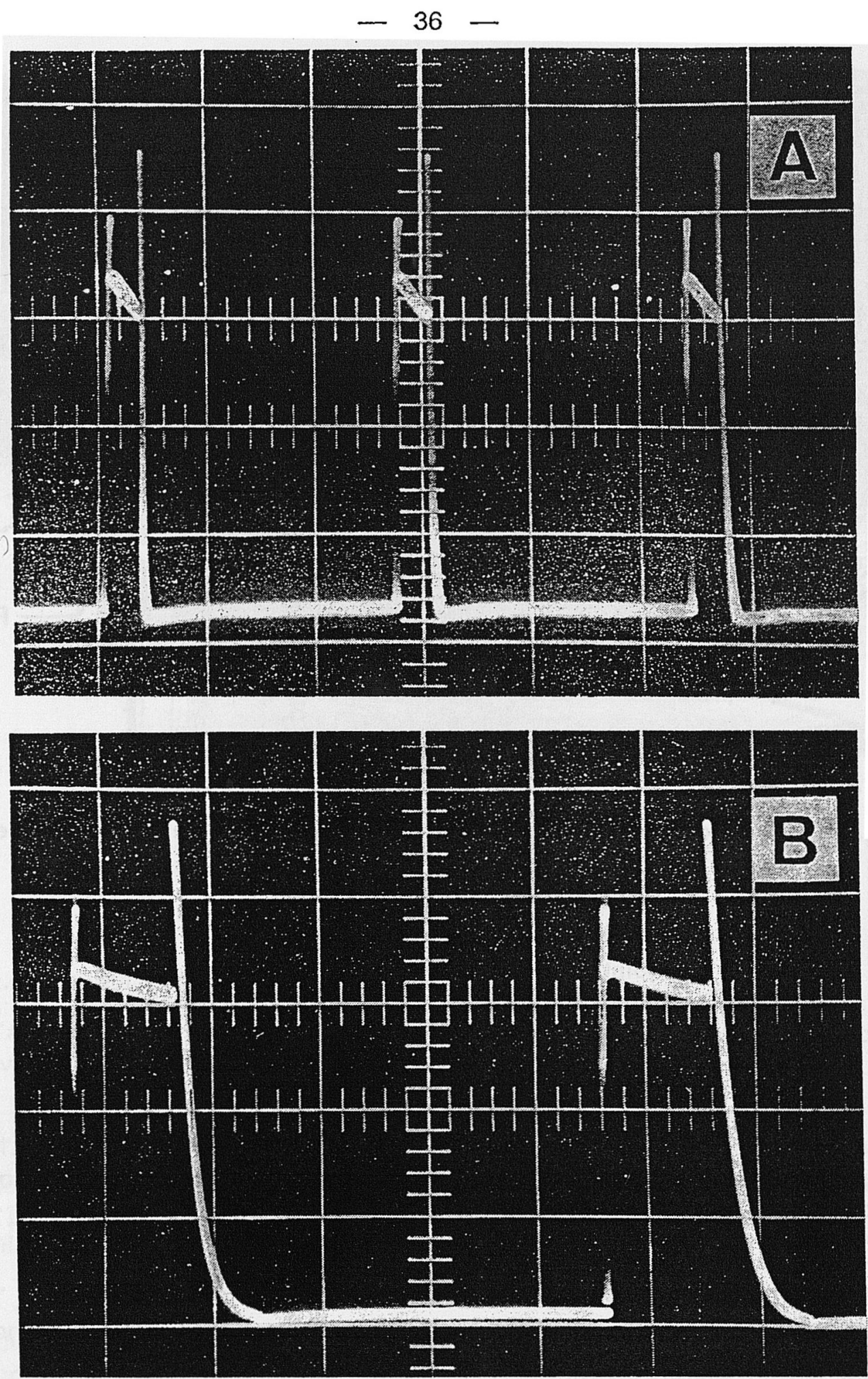

Fig. 3 - Courant en créneaux (fréquence $400 \mathrm{~Hz}$ ).

A. - Conditions limites de fonctionnement :

Charge maximale : $35 \Omega$

Rapport cyclique : $\simeq 10 \%$

Echelle $\left\{\begin{array}{l}\text { temps }: 1 \mathrm{~ms} / \text { carreau } \\ \text { tension }: 50 \mathrm{~V} / \text { carreau. }\end{array}\right.$

B. - Conditions moyennes de fonctionnement :

Charge : $\simeq 100 \Omega$

Rapport cyclique : $25 \%$

Echelle $\left\{\begin{array}{l}\text { temps }: 0,5 \mathrm{~ms} / \text { carreau } \\ \text { tension }: 50 \mathrm{~V} / \text { carreau. }\end{array}\right.$ 


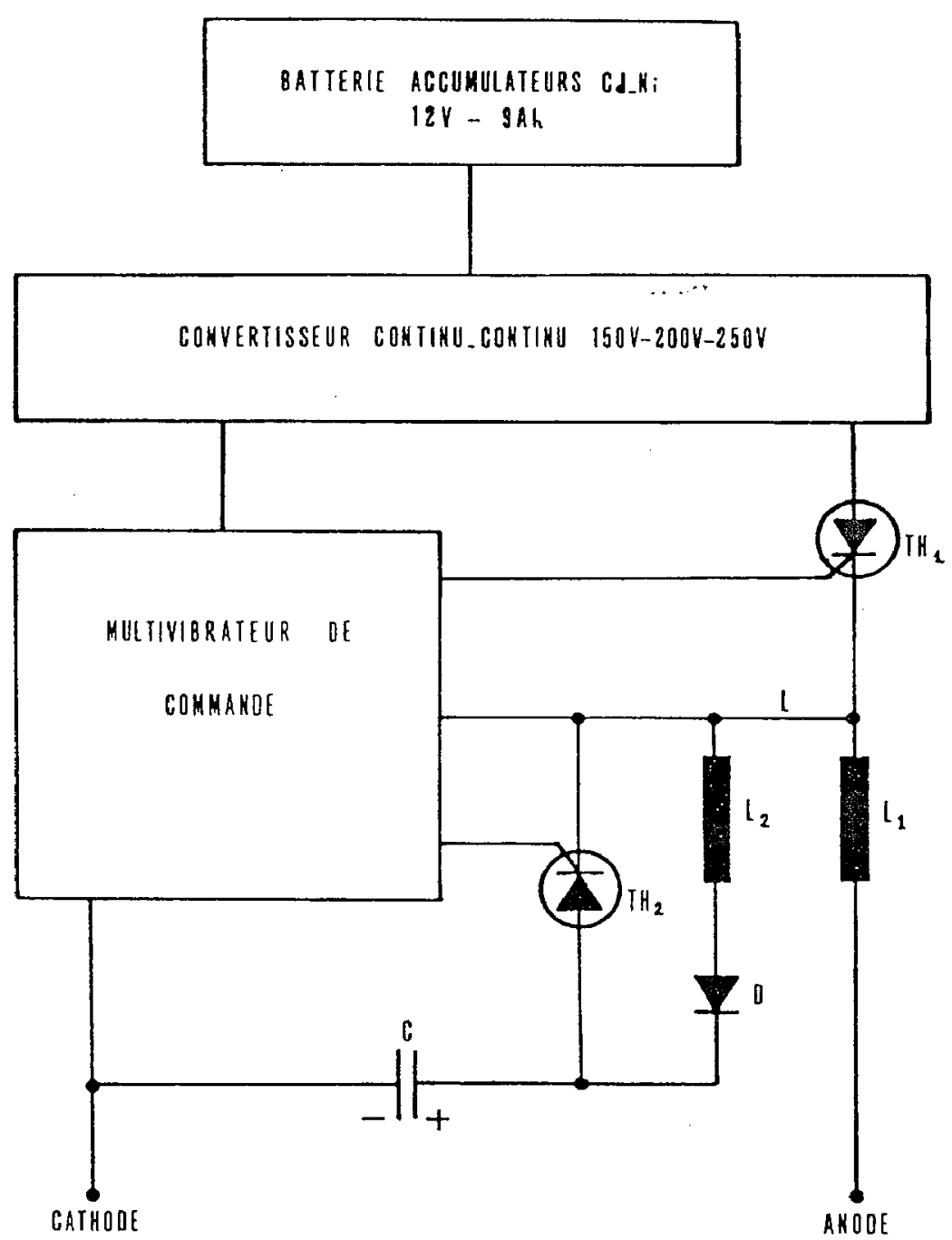

Fig. 4 - Schéma de principe.

l'ensemble un rendement élevé, de l'ordre de $80 \mathrm{p}$. cent et une fréquence fixe de 300 hertz.

Dans ces conditions, la puissance maximale que l'on peut obtenir au secondaire du transformateur est de l'ordre de 100 watts pour les 3 valeurs de la tension de sortie 150,200 et 250 volts.

\subsection{2 - Circuit de commande}

Par un montage multivibrateur astable classique, on obtient des créneaux à fréquence fixe et de largeur variable. Le rapport cyclique peut varier de 10 à 50 pour cent.

Par différenciation de ces créneaux, on obtient des impulsions qui, par l'intermédiaire de transformateurs d'impulsions, parviennent sur les gâchettes des thyristors du "chopper".

L'alimentation du générateur est assurée à partir du secondaire du transformateur dú convertisseur et stabilisée à 24 volts par diode Zener.

\subsection{3 - Chopper à thyristors}

Le montage est alimenté à partir du convertisseur par une tension continue. Lorsque le thyristor $T_{h}$ est amorcé par une impulsion appliquée sur la gâchette, la self $L$ est parcourue dans sa partie $L_{1}$ par le courant traversant $T h_{1}$ et se 
comporte comme un auto-transformateur. Le condensateur $\mathrm{C}$ se charge donc suivant la polarité indiquée, à une tension supérieure à la tẹsion d'alimentation jusqu'à la saturation de la self.

Grâce à la diode $D$, le condensateur $C$ conserve ensuite sa charge jusqu'à ce que le thyristor $\mathrm{Th}_{2}$ soit rendu conducteur. La tension de $\mathrm{C}$ est alors appliquée en inverse sur l'anode de $T h_{1}$ et provoque son désamorçage.

\section{5 - Electrodes (fig. 5)}

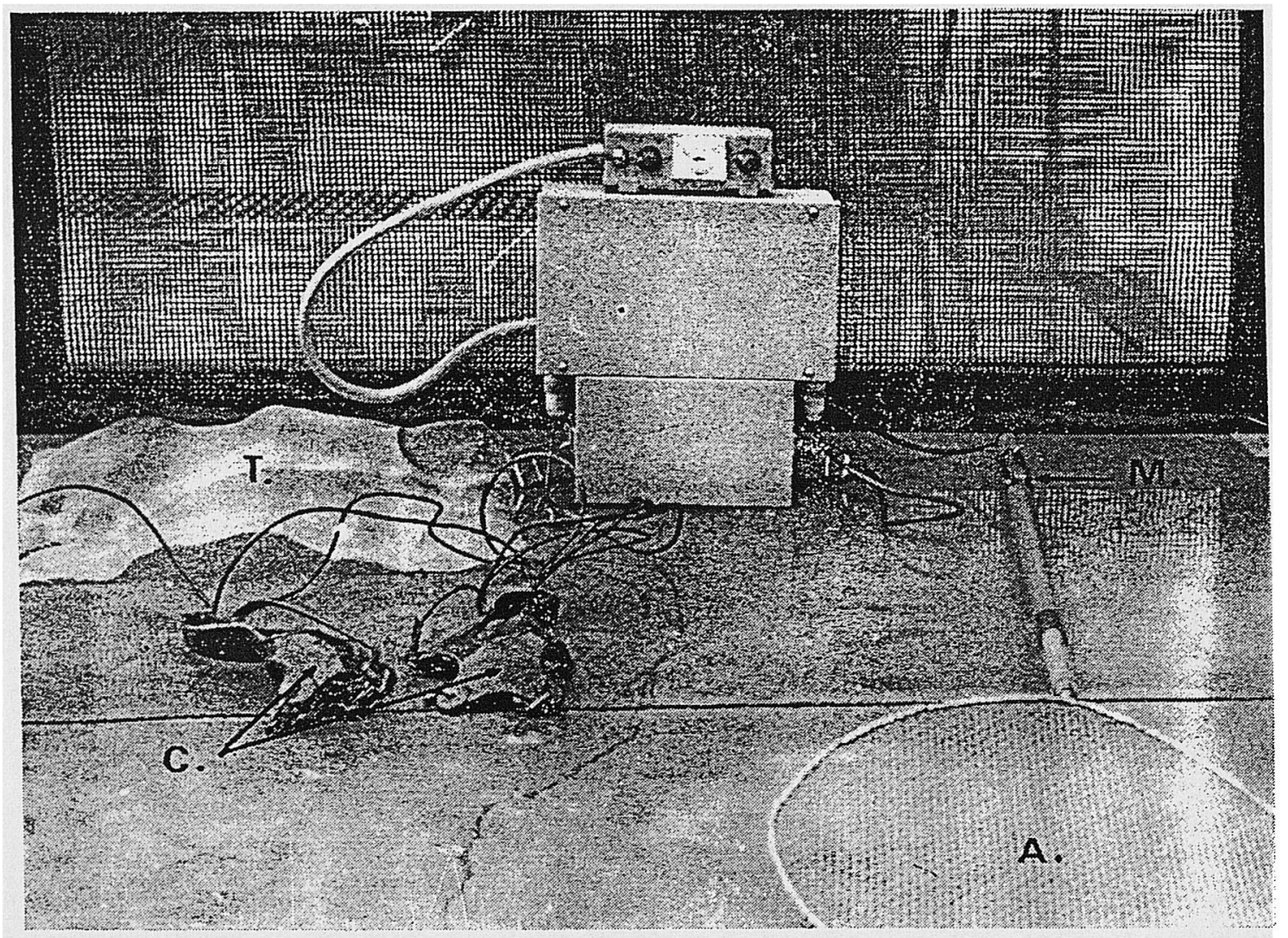

Fig. 5 - Cathode 3 points et anode.

T. : traine

C. : chaînes

A. : anode

M. : minirupteur.

\subsection{1 - Anode}

L'anode est constituée d'un cercle grillagé, amovible, et fixé à l'extrémité d'un manche de canne à pêche télescopique, en fibre de verre. Sur ce manche se trouve un minirupteur basse-tension commandant le courant.

Le cercle grillagé permet éventuellement de ramasser directement les poissons, mais surtout d'assurer une meilleure répartition et une meilleure diffusion du courant dans l'eau, et de jouer un rôle de protection pour le poisson.

L'appareil est livré avec une anode de $30 \mathrm{~cm}$ de diamètre, mais l'utilisateur aura intèrêt à se fabriquer, d'une part, une anode de $40 \mathrm{~cm}$ s'il doit pêcher dans des eaux profondes très résistantes et d'autre part, une anode constituée d'un simple cercle de fil de cuivre $(\varnothing 5 \mathrm{~mm})$ de $30 \mathrm{~cm}$ de diamètre sans grillage pour les eaux conductrices. 


\subsection{2 - Cathode}

La cathode présente trois points de contact avec l'eau :

- 2 chaines anti-dérapantes fixées sous les bottes du pêcheur ;

- 1 *traine" en toile de laiton de $500 \times 500$ millimètres.

Cette cathode " 3 points" permet une meilleure répartition du champ électrique qui se trouve augmenté près de l'anode (utilisée pour la pêche) et diminué près de la cathode.

Les chaines permettent d'assurer un minimum de passage de courant lorsque la traine pour une raison quelconque n'est plus immergée dans l'eau (passage sur un rocher par exemple).

Ce dispositif permet en outre de pêcher à partir de la berge, simplement en immergeant la traine dans l'eau.

\section{6 - Boîtier de commande et de contrôle (fig. 6)}

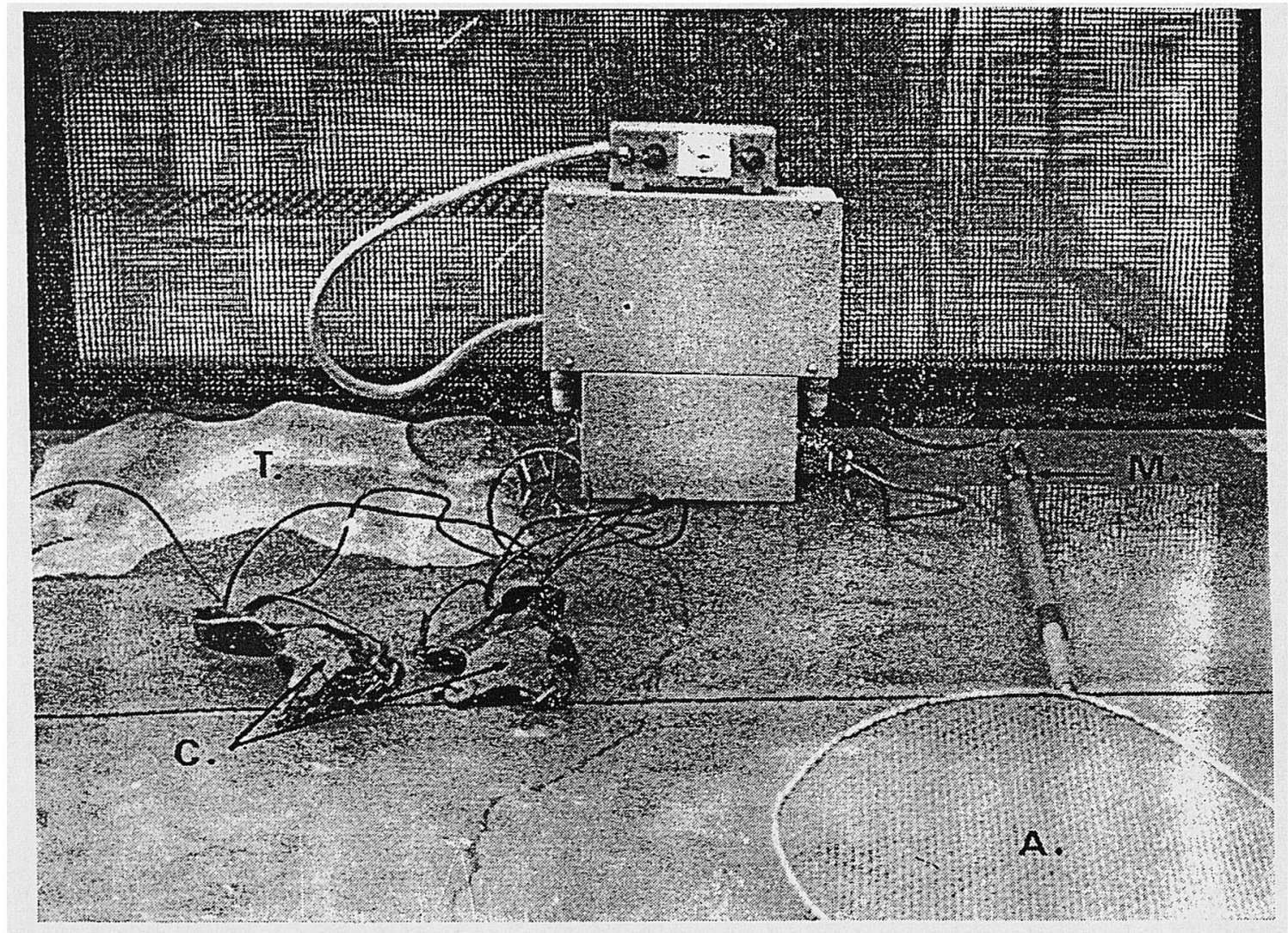

Fig. 6 - Boitier ventral de commande.

R.C. : bouton de réglage du rapport cyclique

position 1 : $10 \%$

position 2 : $20 \%$

position $6: 60 \%$ (sur certains appareils seulement)

T. : bouton de réglage de la tension

position 1: $150 \mathrm{~V}$

position 2: $200 \mathrm{~V}$

position $3: 250 \mathrm{~V}$

I. : interrupteur de sécurité

A. : ampèremètre

F. : fusible de protection. 
Ce boitier permet de régler et de vérifier le fonctionnement de l'appareil en cours de pêche.

II comprend :

- un interrupteur général (1.),

- un bouton de réglage du rapport cyclique (R.C.),

- un bouton de réglage de la tension (T.),

- un ampèremèire (A.) indiquant la consommation de la batterie,

- un fusible de protection (F.) pour l'appareil, en cas de court-circuit.

\section{2. - PERORORATCES}

\section{1 - Autonomie}

En fonctionnement continu, le temps de fêche assuré par la batterie peut varier de $1 \mathrm{~h}$ à 2 h 30 suivant la puissance utilisée et la conductivité de l'eau.

Cependant, grâce à la technique de pêche pratiquée avec le MartinPêcheur (fonctionnement intermittent, voir $\S$. ce temps est normalement porté de $2 \mathrm{~h}$ à $5 \mathrm{~h}$ dans les mêmes conditions.

\section{2 - Efficacité}

Rappelons que l'efficacité est définie par le pourcentage de poissons capturès lors d'une pêche. II existe des efficacités de première, deuxième, troisième pêche...., qui vont en général en décroissant.

D'après des mesures faites dans une douzaine de ruisseaux des PyrénéesAtlantiques, de 3 à $5 \mathrm{~m}$ de largeur et de 0,20 à $0,50 \mathrm{~m}$ de profondeur, l'efficacité de la 1 re pêche varie de:

- 50 à $90 \%$ pour les truites de plus de $14 \mathrm{~cm}$ de longueur,

- 25 à $70 \%$ pour les truitelles de moins de $14 \mathrm{~cm}$ de longueur,

- 50 à $80 \%$ pour les anguilles,

- 30 à $50 \%$ pour les goujons.

Pour les secondes pêches, il faut compter que l'efficacitè est inférieure de $10 \%$ en moyenne.

\section{3 - Limites d'utilisation}

Sans modification de ses accessoires, le Martin-Pêcheur est conçu pour pêcher dans des eaux de résistivité comprise entre 2500 ohms-cm (conductivité $<400$ microsiemens-cm) et 20000 ohms-cm (conductivité $>50$ microsiemens-cm).

Les performances du Martin-Pêcheur peuvent être considérablement augmentées par une modification de ses électrodes.

- Pour des eaux très conductrices (2500 à 1000 ohms-cm), les modifications à apporter par ordre de conductivité croissante sont les suivantes : diamètre :

a) - remplacement de l'anode grillagée par un anneau de cuivre de même 
b) - réduction de la superficie de la traine cathodique, pouvant ailer jusqu'à sa suppression, dans des eaux exceptionnellement conductrices. Dans cette dernière éventualité (suppression), l'efficacité s'en ressentira.

- Pour des eaux très résistantes (20 000 à 50000 ohms-cm), les modifications à apporter par ordre de résistivité croissante sont les suivantes :

a) - augmentation de la surface de la traine cathodique.

b) - augmentation du diamètre de l'anode grillagée.

\section{4 - Sécurité}

Elle est assurée par deux interrupteurs coupant le courant à la batterie.

L'interrupteur du boitier de commande doit être mancuvré chaque fois que, pour une raison ou une autre, la péche est interrompue.

Le minirupteur du manche d'anode commute un relais sous 12 volts : il n'y a don a pas de risque d'électrocution en cas d'isolement défectueux. II ne commute que par pression (douce) : en cas de chute malencontreuse du pêcheur dans l'eau. la pression sur l'interrupteur cessant, le courant est coupé à la batterie.

\section{3. - PRINCIPES D'UTLISATION}

Le Martin-Pêcheur est un appareil portable, ce qui implique une puissance relativement faible : le problème revient à utiliser au mieux cette puissance, en fonction des diverses conditions de pêche imposées par la résistivité et la profondeur du plan d'eau à pêcher.

Ces principes de pêche à pleine puissance ont pour conséquence, en pratique, de limiter la durée de fonctionnement de la batterie à 2 heures. Si l'on c'ésire augmenter cette durée pour des raisons de commodité, on pourra pêcher à fuissance réduite, en utilisant un rapport cyclique inférieur.

Grâce au choix de la fréquence $400 \mathrm{~Hz}$, la baisse d'efficacité ne sera pas très importante.

Les figures 7 et 8 donnent la résistance inter-électrodes en fonction de la résistivité et de la profondeur de l'eau. Ces abaques ont été établis d'après des mesures effectuées avec les électrodes du Martin-Pêcheur (cathode 3 points et anodes griliagées d'un diamètre de 30 et 40 centimètres).

La résistance a été mesurée au moyen d'une batterie de $12 \mathrm{~V}$, par application de la loi d'Ohm, afin d'éliminer les erreurs de mesure dues aux phénomènes de polarisation des électrodes. La résistivité a été mesurée au Pont de Wheastone.

On constate que la résistance inter-électrodes cesse pratiquement de diminuer au-delà de $0,80 \mathrm{~m}$ de profondeur, et que la variation d'amplitude est d'autant plus réduite que la résistivité de l'eau est plus faible.

II s'agit là de valeurs moyennes, la nature du sous-sol provoquant une assez grande dispersion des résultats.

Les abaques de la figure 9 et le tableau 1 permettent de déterminer les conditions optimales de réglage en fonction de la résistance inter-électrodes et de !a profondeur du plan d'eau à pêcher. 


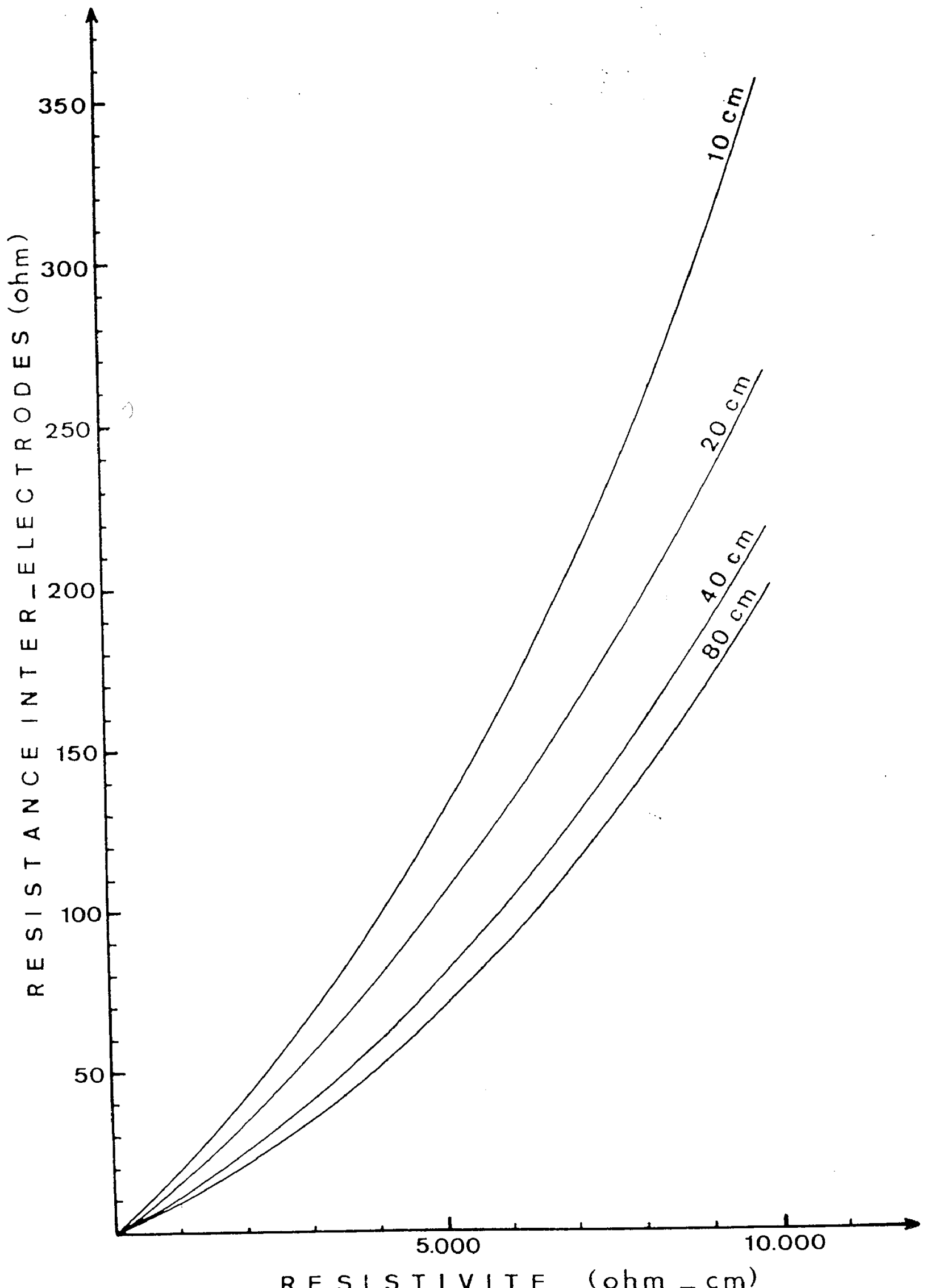

Fig. 7 - PETITE ANODE (diamètre : $30 \mathrm{~cm}$ )

Relation entre résistance inter-électrodes et résistivité en fonction de la profondeur. 


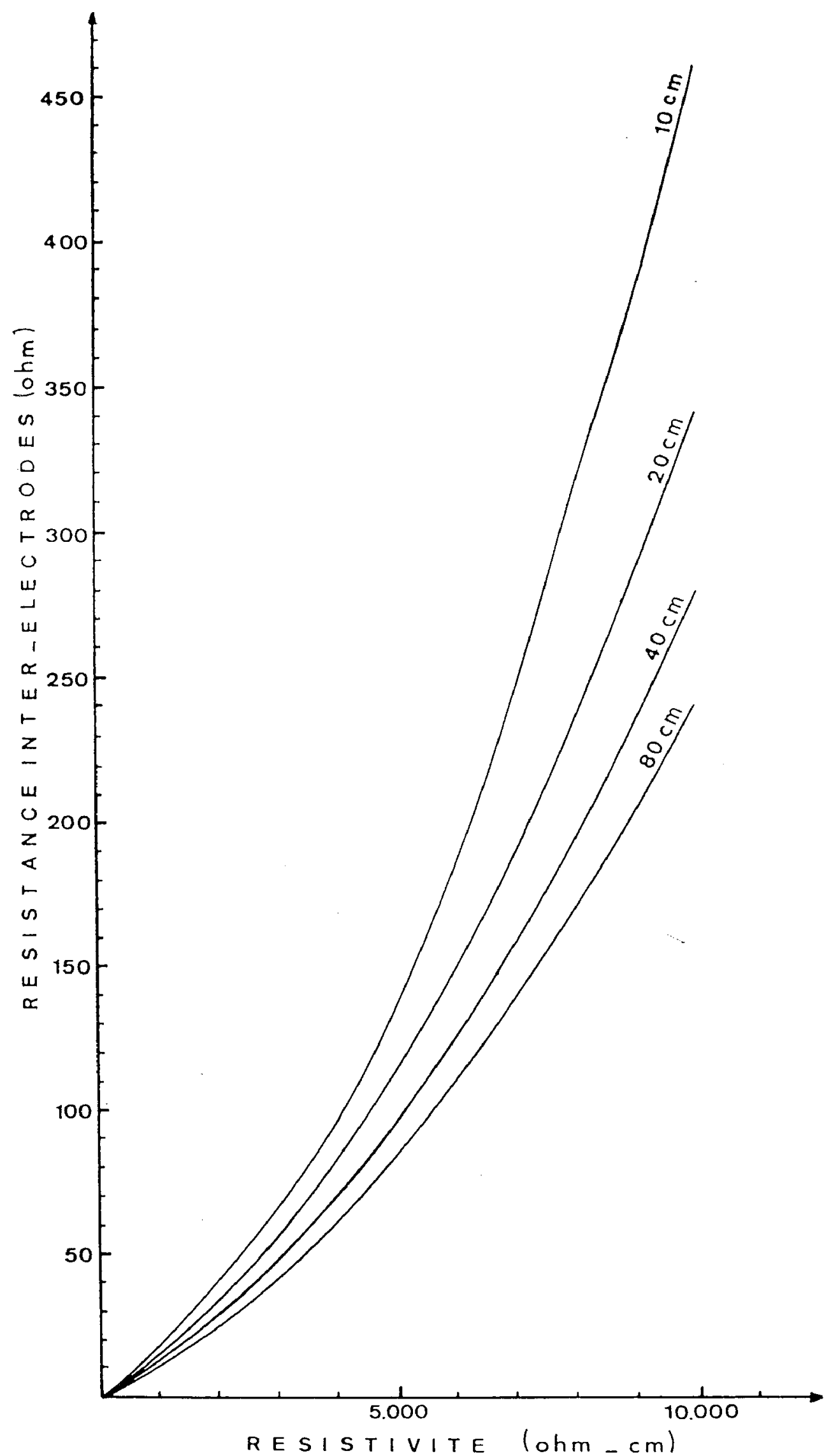

Fig. 8 - GRANDE ANODE (diamètre : $40 \mathrm{~cm}$ ) Relation entre résistance inter-électrodes et résistivité en fonction de la profondeur. 


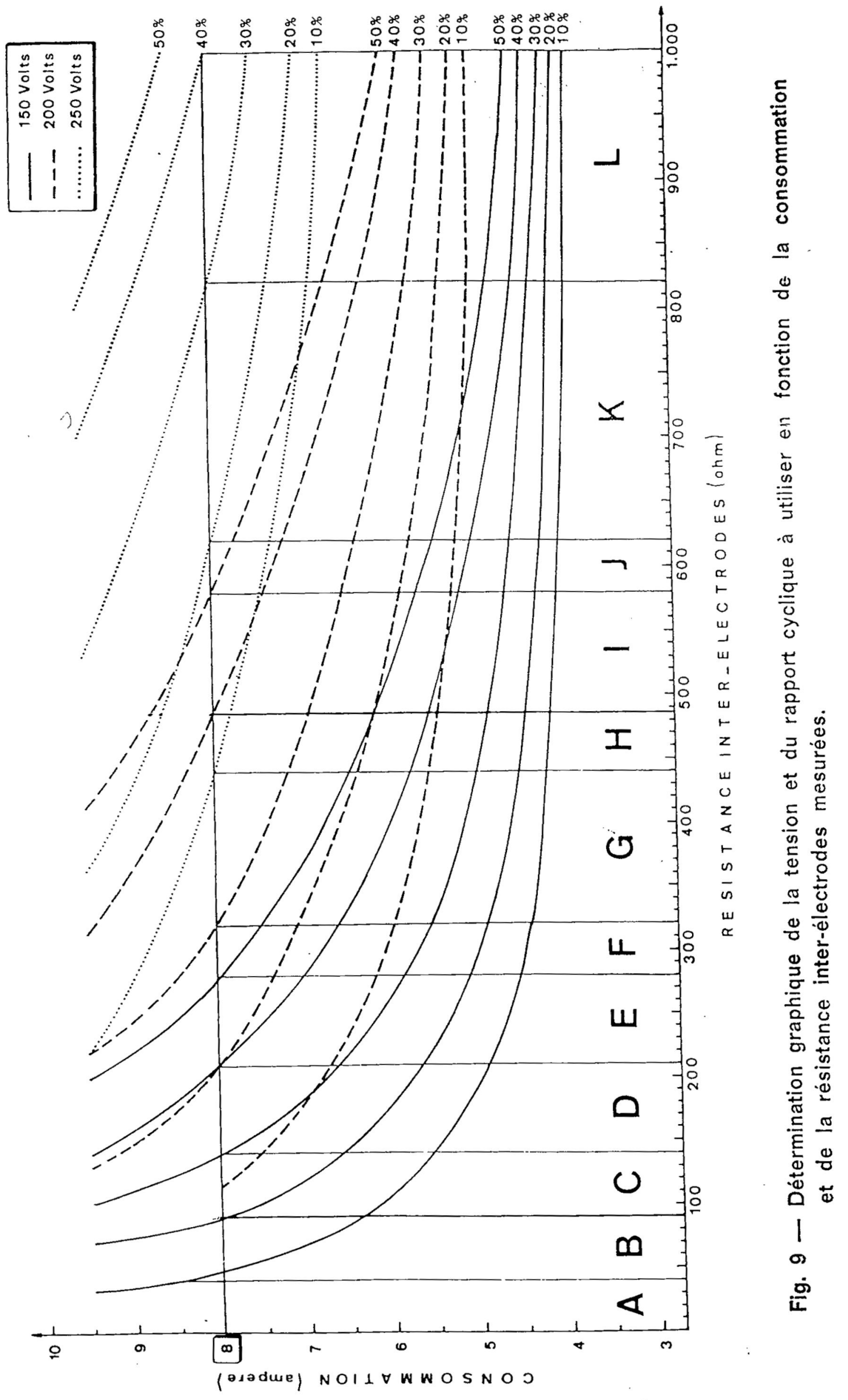




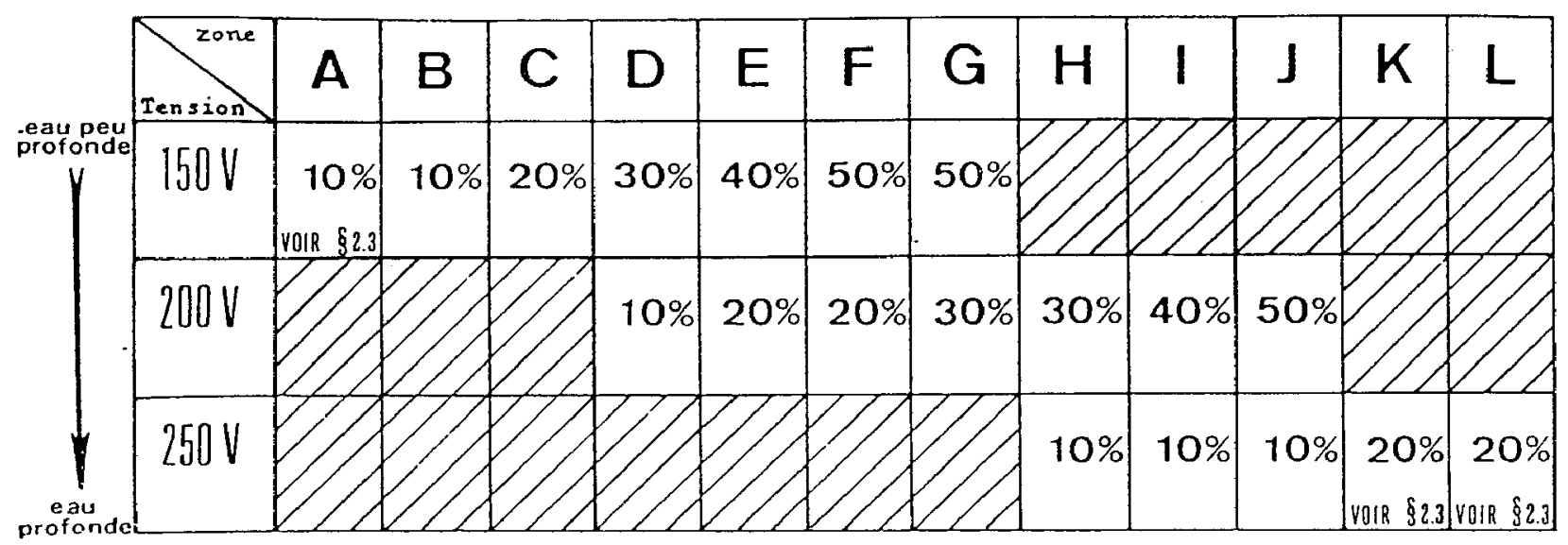

TABLEAU 1 : Tension et rapport cyclique recommandés en fonction de la résistance inter-électrodes et de la profondeur relative de la rivière.

Deux principes directeurs doivent être considérés pour l'obtention d'une efficacité optimale, lorsque l'appareil fonctionne à pleine puissance $(8 \mathrm{~A})$ :

a) - en règle générale, il convient d'utiliser le rapport cyclique maximal et la tension minimale.

Par exemple, pour une résistance inter-électrodes de 210 ohms, on choisira la solution $150 \mathrm{~V}-40 \%$, plutôt que $200 \mathrm{~V}-20 \%$ (Fig. 9).

b) - dans les cas particuliers d'eau profonde, oú il importe d'accroitre la distance d'action du courant, il est préférable de pêcher avec une tension plus forte en réduisant le rapport cyclique (tableau 1). Dans l'exemple pris précédemment, on préfèrera la seconde solution.

\section{4. - PEGLAGE}

Des consignes détaillées sont données dans une notice technique accompagnant l'appareil. Le principe de réglage pour l'obtention d'une efficacité optimale est très simple:

- tout d'abord, l'appareil étant réglé sur les positions correspondant à une tension de 150 volts et un rapport cyclique de $10 \%$. on lit l'indication de l'ampèremètre ;

- puis on se réfère au schéma figurant sur le côté du boitier de commande qui permet de déterminer la tension à utiliser en fonction de l'intensité lue et de la profondeur (fig. 10).

- cette tension étant affichée, on règle le rapport cyclique de manière à obtenir la consommation de courant désirée.

\section{5. - TECHNIQUE DE PECHE}

La technique employée diffère sensiblement de celle pratiquée classiquement avec le courant continu. Dans cette dernière technique, en effet, les électrodes restent constamment sous tension pendant que se pratique la pêche. Avec le Martin-Pêcheur, il est préférable d'interrompre fréquemment le courant, pour prolonger la durée de fonctionnement de la batterie, et pour surprendre les poissons. Le courant étant coupé, on place l'électrode à $40 \mathrm{~cm}$ environ de l'endroit où l'on présume que se trouve le poisson puis on établit le courant. 


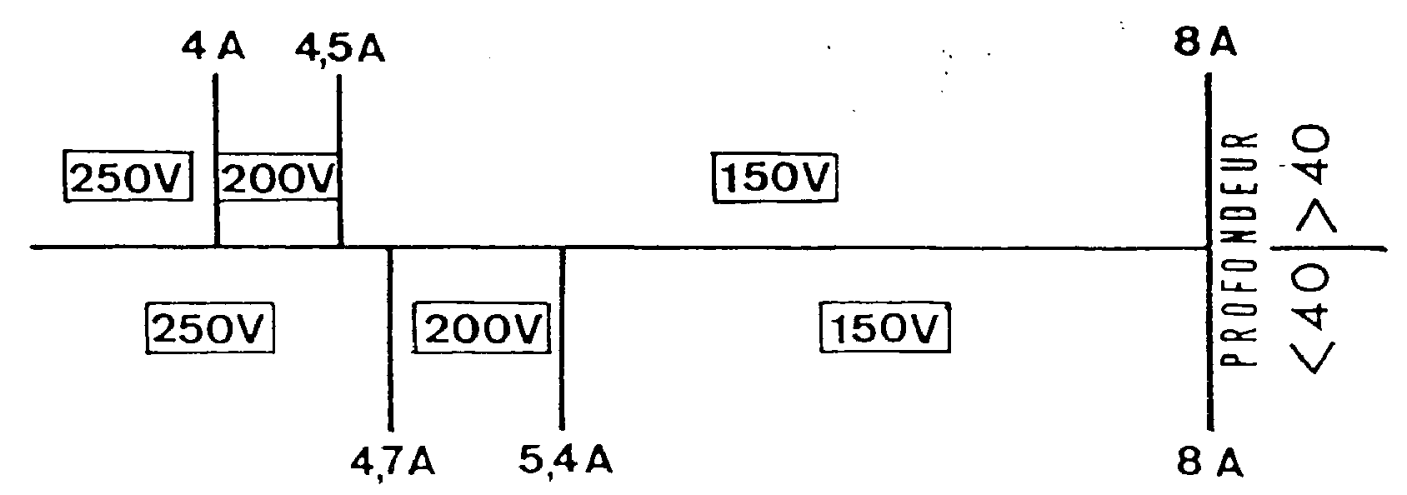

Fig. 10 - Choix de la tension en fonction de l'intensité lue à l'ampèremètre et de la profondeur de la rivière (diagramme figurant sur le côté du boitier de commande).

Lorsque la pêche se révèle difficile (présence de branches par exemple), il est préférable de couper et de rétablir le courant plusieurs fois de manière à permettre le maintien des poissons à proximité de l'anode, sans trop les " choquer.

\section{6. - CONCLUSIONS}

La rapidité de mise en service et les facilités de manipulation du MartinPêcheur en font un appareil très pratique pour les petits cours d'eau à truites. Par rapport à la pêche électrique classique avec groupe électrogène, cet appareil permet de supprimer deux opérateurs : celui qui s'occupe du fil conducteur et celui qui assure la sécurité près du groupe. Ses possibilités d'utilisation se rapprochent de celles d'un groupe à courant continu de 3 kilowatts.

Le Martin-Pêcheur a été particulièrement étudié pour les rivières à truites de petite et moyenne importance mais il peut évidemment être utilisé pour d'autres types d'eau. L'étude complète de son efficacité en fonction des diverses espèces de poissons n'a pas encore été effectuée. 11 convient cependant de souligner déjà son efficacité élevée avec les brochets et brochetons.

Sa limite d'utilisation dans les eaux conductrices est de 1000 ohms-cm. Pour couvrir l'ensemble des eaux continentales, il faudrait un appareil portable permettant de pêcher dans des eaux de résistivité fuérieure à $500 \mathrm{ohms}-\mathrm{cm}$. Des recherches se poursuivent dans ce sens à Biarritz, où est étudiée la réalisation d'un appareil pesant une vingtaine de $\mathrm{kg}$, alimenté par un groupe électrogène de $600 \mathrm{~W}$ et fonctionnant sur le même principe. 